\title{
Composition of phenolics and volatiles in strawberry cultivars and influence of preharvest hexanal treatment on their profiles
}

\begin{abstract}
Biochemical changes of quality-determining components were evaluated in strawberry fruit subjected to preharvest spray treatments using a hexanal-containing formulation that is known to enhance shelf life and quality of fruits. Phenolic compounds and volatiles of fruits of four strawberry cultivars (Mira, Jewel, Kent, and St. Pierre) grown in southern Ontario were characterized by HPLC-MS and solid phase micro extraction (SPME) analysis. Qualitative and quantitative profiles of phenolic compounds varied among the cultivars. In all the cultivars, anthocyanins constituted the most prominent class of phenolic compounds. Volatile profiles of strawberry homogenate differed among the cultivars. Changes in phenolics and volatiles composition were determined in fruits of Mira and Jewel after spraying with a hexanal-containing formulation at weekly intervals. In Jewel, preharvest hexanal spraying altered the profiles of polyphenolic components, while minimal changes were noticed in Mira. Interestingly, very few differences were identified in ester profiles of treated and untreated Mira. In general, hexanal spray application resulted in a decrease in the abundance of several volatile components including esters, ketones, and lactones in treated Jewel compared with the control. The results suggest that cultivar-specific quality changes may result from a preharvest application of hexanal formulations, which may also imply different patterns of metabolite channeling and delay of fruit ripening processes.
\end{abstract}

Keyword: Strawberry; Phenolic compounds; Volatile profiles; Hexanal application 